\title{
Impairment and Rehabilitation in Bilingual Aphasia: A SOM-Based Model
}

\author{
Uli Grasemann ${ }^{1}$, Chaleece Sandberg ${ }^{2}$, Swathi Kiran ${ }^{2}$, and Risto Miikkulainen ${ }^{1}$ \\ ${ }^{1}$ \{uli,risto\}@cs.utexas.edu \\ Department of Computer Science \\ The University of Texas at Austin, Austin, TX 78712, USA \\ 2 challysand@gmail.com, kirans@bu.edu \\ Department of Speech, Language \& Hearing Sciences \\ Boston University, Boston, MA 02215, USA
}

\begin{abstract}
Bilingual aphasia is of increasing interest because a large and growing proportion of the worlds population is bilingual. Current clinical research on this topic cannot provide specific recommendations on which language treatment should focus in a bilingual aphasic individual and to what extent cross-language transfer occurs during or after rehabilitation. This paper describes a SOM-based model of the bilingual lexicon, and reports on simulations of impairment and rehabilitation in bilingual aphasia. The goal is to create computational methods that can complement clinical research in developing a better understanding of mechanisms underlying recovery, and that could be used in the future to predict the most beneficial treatment for individual patients.
\end{abstract}

Keywords: Bilingualism, Aphasia, Lexicon, Neural network, SOM

\section{Introduction}

Aphasia is the partial or complete loss of language function due to brain damage, most commonly following a stroke. In bilinguals, aphasia can affect one or both languages, and during rehabilitation and recovery, the two languages can interact in complex ways. Current research on bilingual aphasia has only begun to inform us about these interactions. At the same time, a better understanding of language recovery in bilinguals is badly needed to inform treatment strategies. Decisions like the choice of a target language for treatment affect the outcome in ways that are currently unpredictable, and the optimal treatment strategy is thought to depend on many factors, including how late the second language was learned and the degree of impairment in either language [26].

The problem of choosing the right treatment approach is of considerable practical importance: Over half the world's population today is bi- or multilingual [16], making bilingual aphasia at least as common as its monolingual counterpart. Moreover, treatment is most effective during a limited time window, and resources available for treatment are often limited. As the proportion of bilinguals in the world increases, so will the potential benefits of more targeted and effective treatment. 
Current clinical research faces considerable difficulties in providing the necessary insight. Too many factors contribute to the outcome of rehabilitation, including which first and second languages (L1 and L2) the patient speaks, the second-language age of acquisition (AoA), the relative pre-stroke competencies, and the relative impairments in both languages. The large number of possible combinations of these factors, and thus of possible treatment scenarios, makes it impractical to examine treatment effects clinically in a systematic way.

In this situation, computational modeling can be a useful tool to complement and guide clinical research. Neural network-based models of impairment and recovery can be used systematically to simulate treatment scenarios and to predict outcomes. These predictions can then inform clinical research, which in turn provides data to validate the model.

This paper reports on recent progress in work that follows this approach. A model of the bilingual human lexicon based on self-organizing maps is trained and then lesioned in order to model lexical access in bilinguals before and after the onset of aphasia. The model is matched to, and compared with, human subject data collected from a group of aphasic patients. Additionally, a simulation of language-based treatment is developed, and is used to investigate a range of treatment scenarios. The treatment simulation makes testable predictions, and could ultimately be used to simulate treatment for individual patients, and to predict the treatment strategy most beneficial in each specific case.

\section{Lexical Access and Bilingual Aphasia}

The mental lexicon, i.e. the storage of word forms and their associated meanings, is a major component of language processing. Lexical access is frequently disturbed in aphasia, and naming impairments are especially common, where patients have trouble recalling words or naming objects (anomic aphasia). The mental lexicon of bilinguals is considerably more complex than that of monolinguals, and the way in which multiple language representations can develop, coexist, and interact in the human brain has an important bearing on our understanding of naming impairment in bilingual aphasia.

Current theoretical models of the bilingual lexicon generally agree that bilingual individuals have a shared semantic (or conceptual) system and that there are separate lexical representations of the two languages. However, the models differ on how the lexica interact with the semantic system and with each other.

The concept-mediation model [25] (Fig. 1a), proposes that both the first (L1) and the second-language lexica directly access concepts. In contrast, the word-association model assumes that second-language words (L2) gain access to concepts only through first-language mediation (Fig. 1b). Empirical evidence [18 suggests that the word association model is appropriate for low-proficiency bilinguals and concept mediation model for high-proficiency bilinguals. As an explanation, De Groot [7. proposed the mixed model (Fig. 1c), where the lexica of a bilingual individual are directly connected to each other as well as indirectly (by way of a shared semantic representation). This model was further revised 
with asymmetry by Kroll \& Stewart [19] (Fig. 1d). The associations from L2 to L1 are assumed to be stronger than those from L1 to L2, and the links between the semantic system and L1 are assumed to be stronger than those between the semantic system and L2.

a

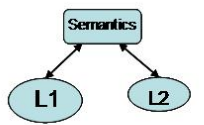

Concept Mediation Mode

$\begin{array}{cc}\text { Concept Mediation Model } & \text { Word Association Mode } \\ \text { (Potter, So, Von Eckardt, \& Feldman, 1984) } & \text { (Potter et al., 1984) }\end{array}$

c

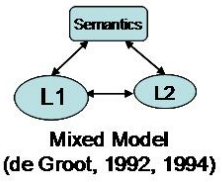

b

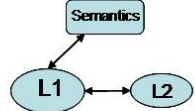

d

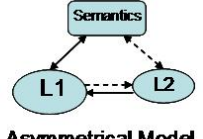

Asymmetrical Model (Kroll \& Stewart, 1994)
Fig. 1. Theoretical models of the bilingual lexicon. All four theories assume a shared semantic system with language specific representations in L1 and L2. The most recent theory (d) includes connections between all maps, with connections of varying strength depending on the relative dominance of the two languages. This theory is used as the starting point for the computational model.

A second important issue is whether activation of the semantic system spreads to both lexica or only within that of the language being used. The prevailing theory suggests that lexical access is target-language nonspecific 4, although, this view is controversial [5]. A third issue is the extent to which proficiency in the two languages and the age at which they are acquired (AoA) affect lexical access. There is evidence that language proficiency, and not AoA, primarily determines the nature of semantic processing 8 .

These issues are of central importance to our understanding of the mechanisms underlying the benefits of language-based treatment in aphasia. Specifically, a kind of treatment that can improve word-finding skills in anomic aphasia is naming treatment, where patients are asked to identify objects or activities shown in pictures [2]. In bilinguals, this treatment can occur in either language, and if lexical access in one language indeed co-activates the other language as well, then that language may be able to recover even if it is not actively used in treatment. The literature on such cross-language transfer is sparse, but several case studies suggest that is does occur [1527, although under what circumstances is currently not known. The computational model described in the next chapter can simulate cross-language transfer, and can potentially help shed light on this and other issues relevant to treatment and recovery.

\section{Modeling Approach}

Although the physiological structure and location of the lexicon in the brain are still open to some debate, converging evidence from imaging, psycholinguistic, computational, and lesion studies suggests that the lexicon is laid out as one or several topographic maps, where concepts are organized according to some measure of similarity 10328 .

Self-organizing maps (SOMs; [16 17]) model such topographical structures, and are therefore a natural tool to build simulations of the lexicon. SOM models 


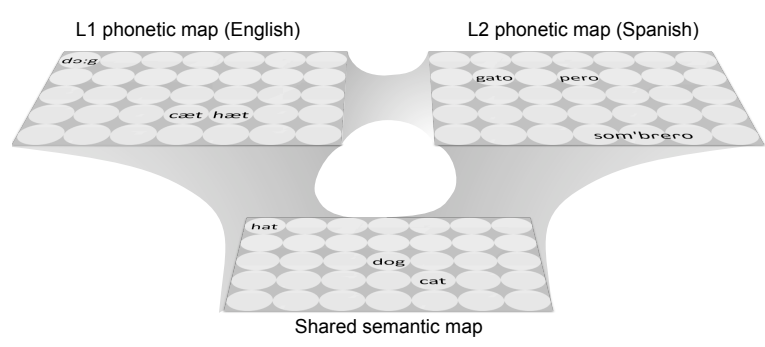

Fig. 2. The DISLEX model is structured after the theoretical model in Fig. 1d. Three SOMs, one each for semantics, L1, and L2, are linked by associations that enable the model to translate between semantic and phonetic symbols, simulating lexical access in bilingual humans.

have been developed to understand e.g. how ambiguity is processed by the lexicon, and how it breaks down in dyslexia 23 , and how the lexicon is acquired during development 21 .

The foundation for the bilingual model used in the present work is DISLEX, a computational neural network model initially designed to understand how naming and word recognition take place in a single language 2223, and later extended to study first language learning [21]. For the purposes of this study, DISLEX was extended to include a second language 24. The resulting computational model, shown in Fig. 2, reflects the revised model by Kroll and Stewart (1994; Fig. 1d): Its three main components are SOMs, one for word meanings, and one each for the corresponding phonetic symbols in L1 and L2. Each pair of maps is linked by directional associative connections that enable network activation to flow between maps, allowing the model to translate between alternative semantic and phonetic representations of a word.

The organization of the three maps and the associations between them are learned simultaneously. Input symbols are presented to two of the maps at the same time, resulting in activations on both maps. Each individual map adapts to the new input using standard SOM training with a Gaussian neighborhood. Additionally, associative connections between the maps are adapted based on Hebbian learning, i.e. by strengthening those connections that link active units, and normalizing all connections of each unit:

$$
a_{i j}^{\prime}=\frac{a_{i j}+\alpha \theta_{i} \eta_{i} \theta_{j} \eta_{j}}{\sum_{k}\left(a_{i k}+\alpha \theta_{i} \eta_{i} \theta_{k} \eta_{k}\right)},
$$

where $a_{i j}$ is the weight of the associative connection from unit $i$ in one map to unit $j$ in the other map, and $\eta_{i}$ is the activation of unit $i$. The neighborhood function $\theta_{i}$ is the same as for SOM training. As a result of this learning process, when a word is presented to the semantic map, the resulting activation is propagated via the associative connections to the phonetic maps, and vice versa. In this way, DISLEX can model both comprehension and production in both L1 and L2.

Note that the L1 and L2 maps have direct connections between them as well, which creates a possible alternative path for the flow of activation between the semantic map (S) and either phonetic map. For example, activation may flow $\mathrm{S} \rightarrow \mathrm{L} 1$ directly, but also $\mathrm{S} \rightarrow \mathrm{L} 2 \rightarrow \mathrm{L} 1$. 
Importantly, such indirect flow of activation between maps can potentially simulate and explain how treatment in one language can benefit the other. For example, if the lexicon is presented with input symbols for S and L1, those maps and the connections between them can be adapted using the method described above. However, in addition, the L2 map is activated indirectly, and that activation can be used to train its associative connections as well. How beneficial this "indirect training" is for L2 may depend on several factors, including the strength and quality of the connections between L1 and L2. The computational experiments reported below will examine this model of cross-language transfer in detail.

The input data used for training the model is based on a list of 300 English nouns gathered for previous studies of naming treatment in aphasia (e.g. 914]). The words were translated into Spanish by a native speaker. Semantic representations are vectors of 261 binary semantic features such as "is a container", or "can be used as a weapon". These features were encoded by hand, and the resulting numerical representations were then used to train the semantic map.

Phonetic representations are based on phonetic transcriptions of English and Spanish words, which were split into spoken syllables, and padded such that the primary stress lined up for all words. The individual phonemes comprising each syllable were represented as a set of phonetic features like height and front-ness for vowels, and place, manner, etc. for consonants [20, similar to the method used in previous work based on DISLEX [23. Phonetic representations consisted of 120 real-valued features for English and 168 for Spanish.

The semantic and phonetic maps of all models were a grid of 30x40 neurons. All learning rates, both for maps and associations, were set to 0.25 . The variance of the Gaussian neighborhood was initially 5 , and decreased exponentially with a halflife of 150 training epochs. Training always lasted 1000 epochs; the number of randomly selected English and Spanish words trained during each epoch was controlled by two "exposure" parameters.

Second-language AoA was simulated by starting training for the L2 phonetic map and its associative connections as soon as the neigborhood size fell below a specific threshold. For example, a thresholds of 0.7 resulted in training beginning at epoch 425 , and a treshold of 5.0 meant it started at epoch 1 .

The resulting models generally have well-organized semantic and phonetic maps. Their naming performance, measured as the percentage of semantic symbols that are translated correctly into their phonetic equivalents, is close to $100 \%$ (98\% for English, 97\% for Spanish) for a wide range of combinations of AoA and exposure. However, as expected, for very low exposure and/or very late AoA, the performance decreases. This is consistent with human language learning, where performance on second-language naming tests tends to be very good, unless the AoA is very late, or exposure to L2 is very limited [11. As an example, Fig. 3 shows a DISLEX system that was trained on a subset of the input data to make the maps easier to visualize. Semantic and L1 maps reflect semantic and phonetic similarity well. In contrast, the L2 map is poorly organized due to the effect of very late acquisition. 


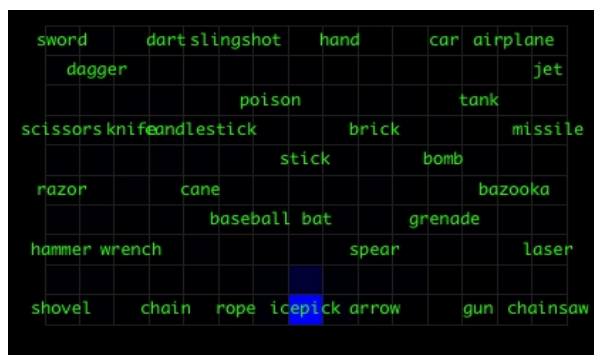

Fig. 3. A DISLEX model trained on the subset of input words with the "can be a weapon" feature. Map activations during a naming task are shown, flowing from the semantic (left) to the English (top right) and Spanish (bottom) maps. Training in Spanish was delayed, leading to a poorly organized map. In this way, the model approximates AoA effects.
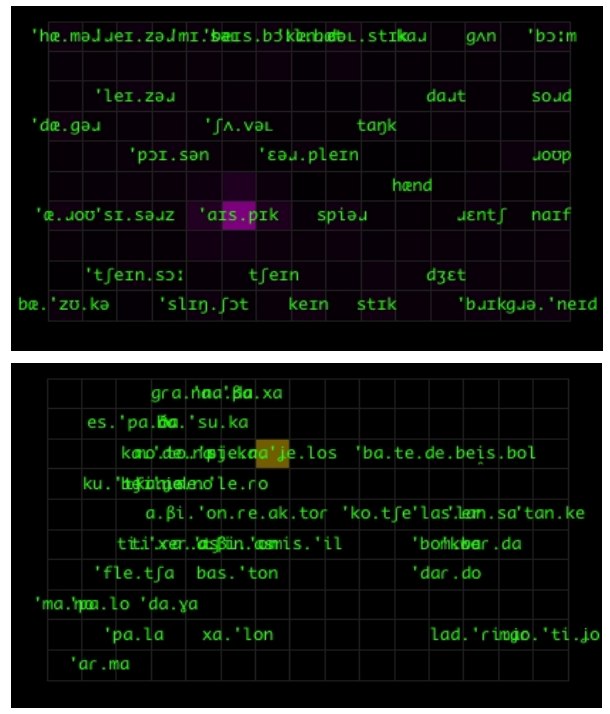

\section{Computational Experiments}

The method to simulate the effects of different levels of exposure and AoAs outlined above was then used to create a number of DISLEX models that were individually tailored to match a group of bilingual patients suffering from aphasia following a stroke. The first step in creating these models was to train DISLEX to match the patients' premorbid state, including naming performance in both Spanish and English, AoA, and exposure data.

Eighteen of the patients were native Spanish speakers, with English AoA varying from from birth to 35 years. One was a native English speaker (AoA 20 years). Premorbid levels of naming performance, AoA, and relative exposure to Spanish vs. English were collected from all patients, and were used to determine the way in which each patient model was trained. The available patient data on language exposure only specified relative exposure (e.g. 30\% Spanish, 70\% English); the absolute amount of exposure was therefore adjusted (retaining the correct ratio) such that the resulting model fit naming performance best.

Fig. 4 shows the language performance of the resulting best-fit models for each patient. Bars show the model's performance; blue triangles are the target data, i.e. the human pre-stroke English and Spanish performance. In most cases $(\sim 80 \%)$, the model is able to match the premorbid language performance (in addition to AoA and relative exposure) of patients well. Why DISLEX sometimes did not achieve a good fit is not clear in all cases. Interestingly, however, at least in one case (\#19), the model identified irregular patient data in this way.

Excluding the patients without a matching DISLEX model, the remaining 16 premorbid models were then used to simulate damage to the lexicon leading to bilingual aphasia. In order to simulate the brain lesion caused by a stroke, the models were damaged by adding increasing levels of Gaussian noise to the associative connections between the semantic and phonetic maps. 
This model of stroke damage was motivated by several known constraints on the mechanisms by which strokes cause aphasia; for example, word comprehension is often relatively spared in aphasia, which could not be simulated in DISLEX using damage to semantic or phonetic maps. Additionally, recent evidence points to white matter damage in anomic aphasia 1213.

Fig. 5 shows how increasing levels of noise damage affect the naming performance of the patient models. The bars on the left side of each plot show the same data as in Fig. 4, i.e. the performance of the undamaged model. Moving from left to right in each plot, the damage increases. Red and green lines show the resulting naming performance in English and Spanish respectively. The vertical position of the triangles pointing left show the patients post-stroke naming performance in English and Spanish, i.e. the performance the damaged models need to match in each case.

By adjusting the amount of damage for English and Spanish separately, each patient's post-stroke naming performance can always be matched, as shown in the figure. Interestingly, however, in all but three cases (81\%), the patient's post-stroke performance can be simulated by damaging English and Spanish connections equally. This is consistent with the type of impairment seen in aphasia patients, which usually, but not always, affects both languages equally. An interesting and counterintuitive prediction of the model is that less proficient languages are more vulnerable to damage than highly proficient ones. This is clearly visible e.g. in models \#1, 3, and 12 .

In the future, these individual models will be used to investigate and predict treatment effects in human patients. As a first step towards this goal, DISLEX simulations for a range of 64 different treatment scenarios were created, which differed in L1 (English/Spanish), AoA (early/late), exposure to L1 and L2 (low/high), damage to L1 and L2 (low/high), and treatment language (English/Spanish). Treatment was simulated by retraining a subset of the original input words in the treatment language. Associative connections of the untreated language were also trained, using indirect activation in the way described in Section 3 .

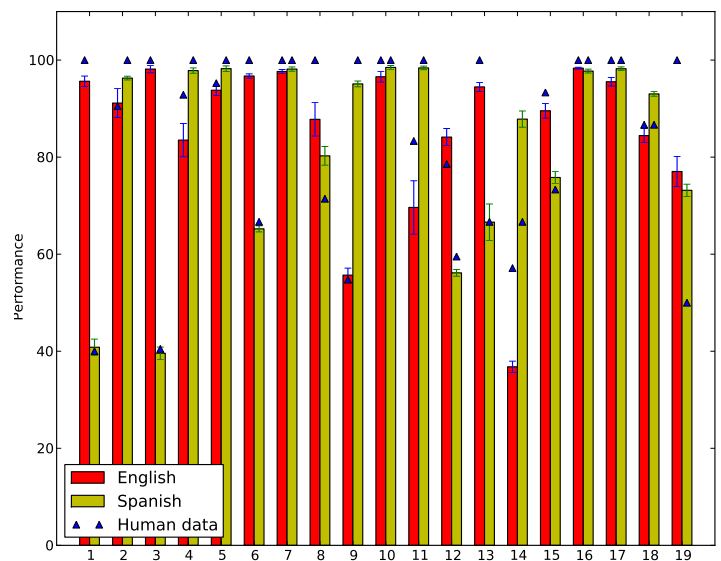

Fig. 4. Modeling the pre-stroke state of bilingual aphasics. The performance of best-fit DISLEX models in English (red bars) and Spanish (yellow bars) is compared to patient data (blue triangles). The models were trained with the same relative exposure as patients to both languages; AoAs were simulated by variably delaying L2 training. The models are generally able to match the patient data well, providing a basis for simulations of stroke damage. 


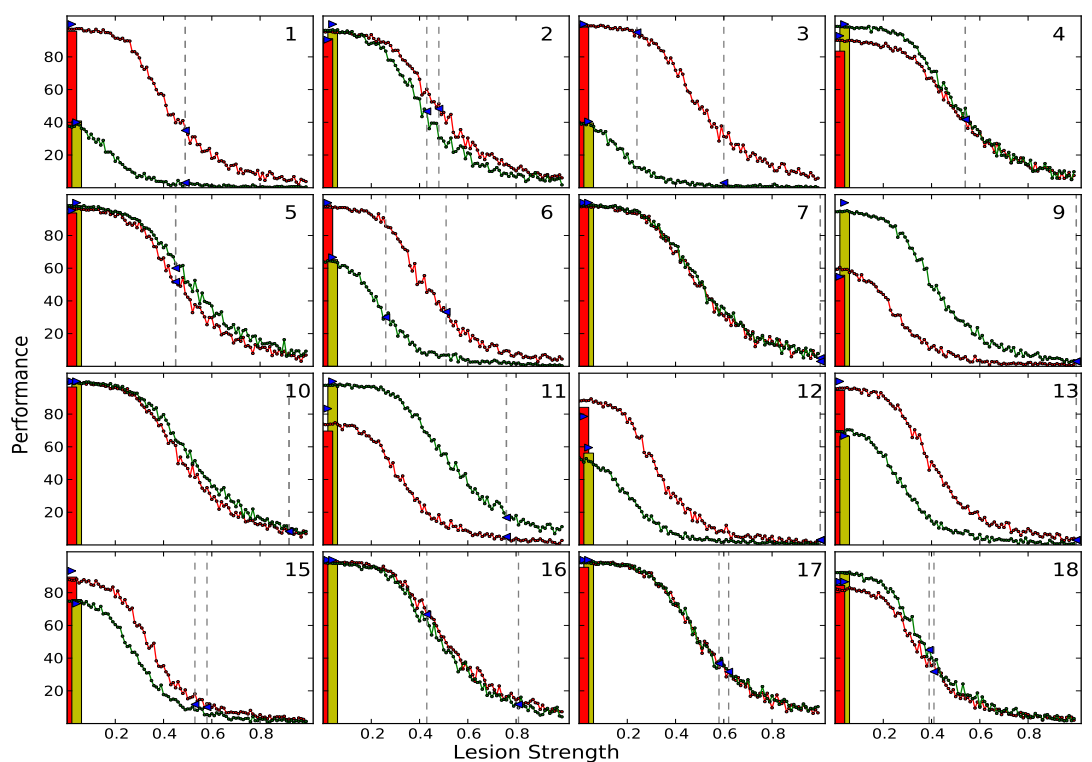

Fig. 5. The effect of damage to the associative connections on naming performance. Patients are numbered as in Fig 4 , bars on the left show the pre-stroke (undamaged) models. Moving from left to right in each plot, damage increases, and performance in English (red line) and Spanish (green line) drops. Triangles pointing left indicate human post-stroke (aphasic) performance, which can in most cases be matched using approximately equal damage to both languages, suggesting that stroke damage is modeled realistically.

Fig. 6 illustrates the clearest prediction of this model of treatment: If one language is damaged more than the other, training the less damaged language benefits the more damaged language, but not vice versa. Surprisingly, all other factors, including relative proficiency and AoA, have little or no effect on crosslanguage transfer in the model. Moreover, the current model predicts that treating one language should benefit the other in the majority of training scenarios independent of treatment language. However, damage in the model was only applied to semantic $\rightarrow$ phonetic connections, and damage to other connections, which may be common in humans, may prevent this in many cases. Future work will investigate such additional damage, which will lead to further testable predictions.

\section{Discussion and Future Work}

In this paper, a bilingual version of DISLEX, a SOM-based model of the human lexicon, was used to simulate impaired lexical access and the effects of languagebased treatment in patients with bilingual aphasia. The model was trained and then lesioned to simulate lexical access before and after the onset of aphasia. Human subject data collected from real aphasic patients was used to demonstrate that the model can account for AoA and exposure effects, and that brain damage 

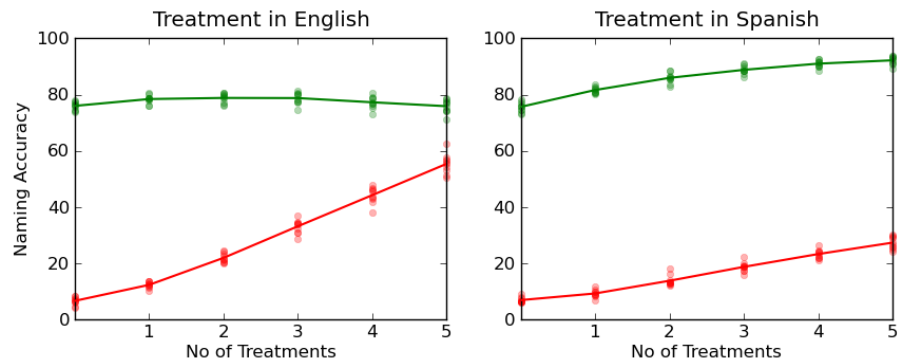

Fig. 6. Effects of treatment language on outcome in the model. Red lines are English, green lines Spanish performance. In the scenario shown, English is L2 (early

AoA), exposure to both languages is low, and English is damaged more than Spanish. The model predicts that treating the less damaged language (in this case Spanish) benefits the more damaged language, but not vice versa.

underlying aphasia can be simulated with a simple noise lesion. Additionally, a simulation of language-based treatment was applied to a range of possible treatment scenarios, and used to predict how the choice of treatment language affects the outcome.

The model makes sevaral testable predictions. First, the effects of noise damage on naming accuracy suggest that in most cases, the brain damage underlying aphasia, not just the impairment, is close to equal for both languages. Second, it predicts that the weaker language, whether it is the first or second, is less resistent to damage than the stronger one. Finally, and most interestingly, the treatment model predicts that using the less damaged language for treatment benefits the more damaged one, but not vice versa.

In future work, the treatment simulation will be applied to the individual patient models, and the results will be compared with the real treatment outcomes. If validated in this way, the model could be used to meaningfully predict the benefits of different treatment approaches, and could ultimately contribute to the development of optimized treatment strategies tailored to individual patients.

\section{References}

1. T. K. Bhatia and W. C. Ritchie, eds., The Handbook of Bilingualism, Blackwell Publishing (2005).

2. M. Boyle and C. Coelho, Application of semantic feature analysis as a treatment for aphasic dysnomia, American Journal of Speech-Language Pathology, 94-98 (1995).

3. A. Caramazza, A. Hillis, E. Leek, and M. Miozzo, The organization of lexical knowledge in the brain: Evidence from category- and modality-specific deficits, in: Mapping the Mind, L. Hirschfeld and S. Gelman, eds., Cambridge University Press (1994).

4. A. Costa, W. Heij, and E. Navarette, The dynamics of bilingual lexical access, Bilingualism: Language and Cognition, 9:137-151 (2006).

5. A. Costa, M. Miozzo, and A. Caramazza, Lexical selection in bilinguals: Do words in the bilingual's two lexicons compete for selection?, Journal of Memory and Language, 43:365-397 (1999).

6. D. Crystal, English as a global language, Cambridge University Press (1997).

7. A. de Groot, Determinants of word translation, Journal of Experimental Psychology: Learning, Memory and Cognition, 18:1001-1018 (1992). 
8. L. Edmonds and S. Kiran, Lexical selection in bilinguals: Do words in the bilingual's two lexicons compete for selection., Aphasiology, 18:567-579 (2004).

9. L. A. Edmonds and S. Kiran, Effect of semantic naming treatment on crosslinguistic generalization in bilingual aphasia, Journal of Speech Language and Hearing Research, 49(4):729-748 (2006).

10. M. Farah and M. Wallace, Semantically bounded anomia: Implications for the neural implementation of naming, Neuropsychologia, 30:609-21 (1992).

11. A. Hernandez and P. Li, Age of acquisition: Its neural and computational mechanisms, Psychological Bulletin, 133:638-650 (2007).

12. J. Fridriksson et al., Impaired speech repetition and left parietal lobe damage, The Journal of Neuroscience, 30(33):1105711061 (2010).

13. J.M. Anderson et al., Conduction aphasia and the arcuate fasciculus: A reexamination of the wernickegeschwind model, Brain and Language, 70:1-12 (1999).

14. S. Kiran, Typicality of inanimate category exemplars in aphasia treatment: Further evidence for semantic complexity, Journal of Speech Language and Hearing Research, 51(6):1550-1568 (2008).

15. K. Kohnert, Cognitive and cognate-based treatments for bilingual aphasia: a case study, Brain and Language, 91(3):294-302 (2004).

16. T. Kohonen, Self-organized formation of topologically correct feature maps, Biological Cybernetics, 43:59-69 (1982).

17. T. Kohonen, Self-Organizing Maps, Springer, Berlin (2001).

18. J. Kroll and J. Curley, Lexical memory in novice bilinguals: The role of concepts in retrieving second language words, in: Practical Aspects of Memory, Vol. 2, Wiley, New York, NY (1988).

19. J. F. Kroll and E. Stewart, Category interference in translation and picture naming: Evidence for asymmetric connections between bilingual memory representations, Journal of Memory and Language, 33:149-174 (1994).

20. P. Ladefoged, Vowels and consonants: An introduction to the sounds of languages, Blackwells, Oxford (2001).

21. P. Li, X. Zhao, and B. MacWhinney, Dynamic self-organization and early lexical development in children, Cognitive Science, 31:581-612 (2007).

22. R. Miikkulainen, Subsymbolic Natural Language Processing: An Integrated Model of Scripts, Lexicon, and Memory, MIT Press, Cambridge, MA (1993).

23. R. Miikkulainen, Dyslexic and category-specific impairments in a self-organizing feature map model of the lexicon, Brain and Language, 59:334-366 (1997).

24. R. Miikkulainen and S. Kiran, Modeling the bilingual lexicon of an individual subject, in: Proceedings of the Workshop on Self-Organizing Maps (WSOM'09), Springer, Berlin (2009).

25. M. Potter, K. So, B. von Eckardt, and L. Feldman, Lexical and conceptual representation in beginning and proficient bilinguals, Journal of Verbal Learning and Verbal Behavior, 23:23-38 (1984).

26. P. Roberts and S. Kiran, Assessment and treatment of bilingual aphasia and bilingual anomia, in: Speech and Language Disorders in Bilinguals, A. Ramos, ed., 109-131, Nova Science Publishers, New York: NY (2007).

27. S. Sasanuma and H. Suk Park, Patterns of language deficits in two korean-japanese bilingual aphasic patients - a clinical report, in: Aspects of bilingual aphasia, M. Paradis, ed., 111-122, Pergamon, Oxford (1995).

28. M. Spitzer, U. Kischka, F. Gückel, M. E. Bellemann, T. Kammer, S. Seyyedi, M. Weisbrod, A. Schwartz, and G. Brix, Functional magnetic resonance imaging of category-specific cortical activation: Evidence for semantic maps, Cognitive Brain Research, 6:309-319 (1998). 